\title{
Performance of the CMS High-Level Trigger
}

\author{
Edgar Carrera Jarrin* \\ (for the CMS Collaboration) \\ Boston University, Boston, MA, USA \\ E-mail: ecarrera@cern.ch
}

The CMS trigger system has been designed to cope with unprecedented luminosities and accelerator bunch-crossing rates of up to $40 \mathrm{MHz}$ at LHC. The High-Level-Trigger (HLT) combines, in a novel way, the traditional Level-2 and Level-3 trigger components, which are implemented in a commercial Filter Farm with thousands of CPUs. The flexibility of a uniform software environment, accessing the full Level-1 rate (100 KHz), allows the coherent tuning of the HLT algorithms to accommodate multiple physics channels and enhance the CMS physics reach. We report on the trigger commissioning of the HLT with the first LHC $p p$ collisions at $7 \mathrm{TeV}$ and discuss the first results on the trigger performance.

35th International Conference of High Energy Physics

July 22-28, 2010

Paris, France

${ }^{*}$ Speaker. 


\section{Introduction}

The Compact Muon Solenoid (CMS) [1] is one of the general purpose experiments of the CERN's Large Hadron Collider (LHC). It consists of an inner pixel and silicon strip tracker system followed by an electromagnetic calorimeter (ECAL) with lead tungstate crystal technology, and a brass-scintillator hadronic calorimeter (HCAL). These sub-systems are enclosed in a superconducting solenoid of $3.8 \mathrm{~T}$ field strength. Outside the solenoid magnet sits a muon spectrometer, which is layered with a steel return yoke interleaved with stations of drift tubes, cathode strip chambers, and resistive plate chambers. The detector is separated in central (barrel) and forward (endcap) regions.

At the design level, the CMS data acquisition system (DAQ) is expected to process information at a bunch-crossing rate of $40 \mathrm{MHz}$ with an instantaneous luminosity of $10^{34} \mathrm{~cm}^{-2} \mathrm{~s}^{-1}$. In order to cope with the high rate, the DAQ uses a two-stage trigger system, the Level-1 (L1) trigger, which is built of custom-made hardware and reduces the rate to $100 \mathrm{KHz}$, and the High Level Trigger (HLT) system $[2,3]$, which further reduces the rate $(O(1000))$ by running offline-quality reconstruction algorithms in a single processor Filter Farm with $\sim 5000$ CPUs. The HLT algorithms are executed in parallel (when possible) through different trigger "paths" that together form the trigger "menu".

During the start-up period of first $p p$ collisions in Spring 2010, the HLT system ran at a maximum input rate of $50 \mathrm{KHz}$ with a maximum instantaneous luminosity of $\sim 5 \times 10^{29} \mathrm{~cm}^{-2} \mathrm{~s}^{-1}$. Plots and a brief description of its excellent performance are presented in these proceedings.

\section{Physics Triggers Performance}

\subsection{Electron and Photon Triggers}

Photons and electron paths share a common step where energy clusters in the ECAL are spatially matched to low-level candidates from L1 decisions. In order to recover energy from bremsstrahlung or photon conversions, groups of clusters (super-clusters) along a road in the azimuthal direction of the detector are constructed. A threshold on the transverse energy $\left(E_{T}\right)$ of the super-cluster is then applied for those super-clusters whose shape is consistent with that of an electromagnetic object and that have calorimetric (ECAL and HCAL) isolation.

For electrons paths, super-clusters are matched to hit pairs in the pixel detector and the reconstruction of a track, using a Kalman Filter, is attempted. Tracks are seeded from pairs of hits in the pixel layers located within a certain spatial region around the direction of the reconstructed electron or photon (regional reconstruction). Finally, loose track isolation in a hollow cone is required for electrons and a threshold on the number of tracks within a cone around the super-cluster direction is enforced for photons.

Figure 1 shows the performance plots for photon triggers on offline reconstructed superclusters and electrons, and the electron pixel matching efficiency.

\subsection{Muon Triggers}

L1 muon candidates are used to seed the reconstruction of tracks in the muon chambers. At the first stage in the HLT, an improved reconstruction of the local muon tracks is attempted based on Kalman-filter techniques and a threshold on the transverse momentum $\left(p_{T}\right)$ is applied. At a second 

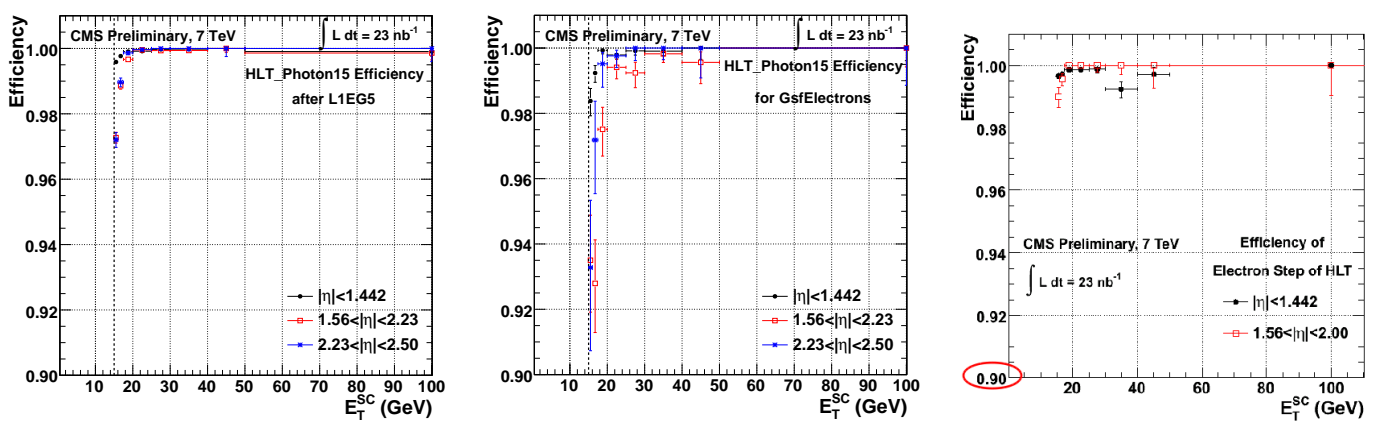

Figure 1: Trigger efficiency for selected offline super-clusters matched to L1 objects to pass a photon trigger with a threshold of $E_{T}>15 \mathrm{GeV}$ (left) and for selected offline electrons matched to L1 objects to pass a photon trigger with a similar threshold (center), plotted vs. the super-cluster $E_{T}$. The efficiency for offline reconstructed electrons, which have passed a photon trigger with a threshold of $E_{T}>15 \mathrm{GeV}$, to pass an electron trigger with a similar threshold as a function of the super-cluster $E_{T}$ and pseudo-rapidity is also shown (right).

stage, the first-stage objects are matched to charged particle tracks reconstructed in the tracking system. The $p_{T}$ resolution is significantly improved after the latter step. Finally, calorimetric isolation in the first stage, and tracker-based isolation in the second step is optionally applied. Figure 2 shows an example of performance plots in the barrel and the endcap regions of the detector.
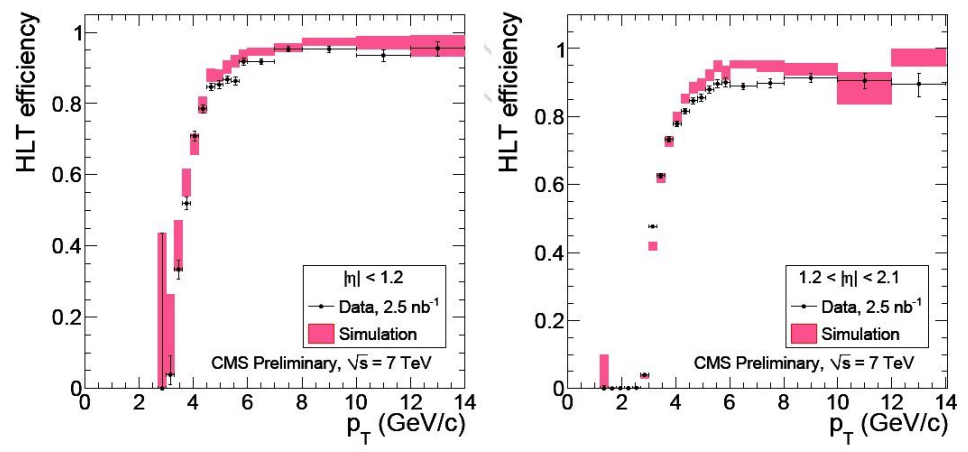

Figure 2: Efficiency for a high-quality offline reconstructed muon to pass the HLT single muon trigger with a threshold of $p_{T}>3 \mathrm{GeV}$, plotted as a function of $p_{T}$ for the barrel (left) and the endcaps (right). Events were collected with the Minimum Bias trigger. Results from MC simulation are also shown; the lower-than-expected efficiency in data is due to time calibration at start-up.

\subsection{Jet and Missing Transverse Energy Triggers}

The jet algorithm takes as input calorimeter "towers" (HCAL cells and corresponding projections on ECAL crystals) with energy deposits above certain $p_{T}$ threshold. An iterative cone algorithm with a cone of radius $R=\sqrt{(\Delta \phi)^{2}+(\Delta \eta)^{2}}=0.5$ is used to reconstruct jets. Missing transverse energy ( $E_{T}^{\text {miss }}$ or MET) is calculated as an algebraic sum of transverse energies of 
calorimeter objects plus muons. $E_{T}^{\text {miss }}$ can be used also in combination with one or more jet requirements.

Figure 3 shows some examples of performance plots for jet/MET triggers.
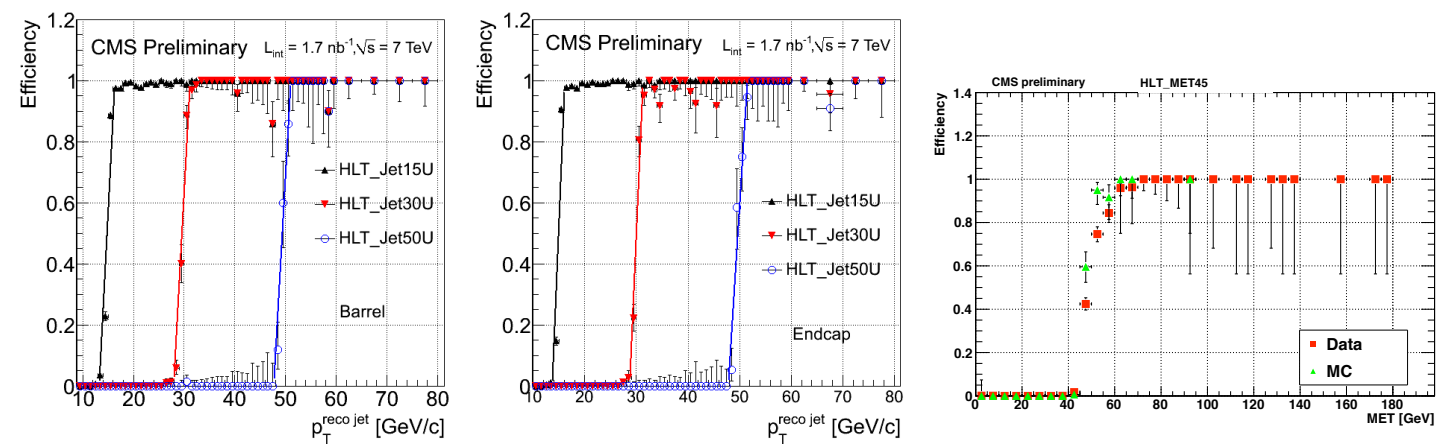

Figure 3: Efficiency for offline reconstructed jets to pass HLT jet triggers of different $p_{T}$ thresholds, namely 15,30 , and $50 \mathrm{GeV}$, plotted versus $p_{T}$, for the barrel (left) and endcaps (center). The efficiency for events passing an $\mathrm{L} 1$ trigger of threshold $E_{T}^{\text {miss }}>20 \mathrm{GeV}$ to pass an HLT trigger with a threshold of $E_{T}^{\text {miss }}>45 \mathrm{GeV}$ and its comparison with $\mathrm{MC}$ simulations is also shown (right).

\section{Trigger Rates and CPU Performance}

An accurate prediction of trigger rates is an essential component for design and trigger metric. Two methods are used to estimate such rates. The first one relies on Monte Carlo (MC) simulation of minimum bias events, and the second one on extrapolation of rates from data. A comparison between the predicted and observed rates is shown in Figure 4. Most trigger rates are predicted accurately within $20 \%$ by both methods. The trigger paths for which there is noticeable disagreement between the predicted and the observed rate have a large noise or cosmic rays contamination.

Another important requirement for the design and monitoring of the HLT is its CPU timing performance. In order not to introduce dead time in the DAQ system, the HLT software is required to make a decision within $100 \mathrm{~ms}$ on average when running with an L1 input rate of $\sim 50 \mathrm{~Hz}$. To measure the timing, the HLT menu is run on a test-stand equipped with machines that are identical to the ones used in the HLT Filter Farm. These machines have 2 Quad Core Intel Xeon 5430 processors with a nominal frequency of $2.66 \mathrm{GHz}$ and $16 \mathrm{~GB}$ of memory.

The data used to make the measurement were collected at an instantaneous luminosity of $\sim 5 \times 10^{29} \mathrm{~cm}^{-2} \mathrm{~s}^{-1}$ with an L1 input rate of $45 \mathrm{KHz}$. The blue histogram in Figure 4 shows the total time per event for the full HLT menu that was used for data taking. The red histogram shows the CPU performance without some special trigger paths that execute full pixel reconstruction. These paths, which were temporarily deployed at start-up luminosities, dominate the entries in the tail of the timing distribution and are used to study particle correlations in high multiplicity events. In both distributions, the calculated mean and RMS include the overflow events. The peak near $20 \mathrm{~ms}$ represents the most probable time for HLT processing, for the given data conditions and trigger menu. The bulk of the distribution is dominated by paths which are either for commissioning and have very few modules, or pass-through paths of L1-accepted events. 

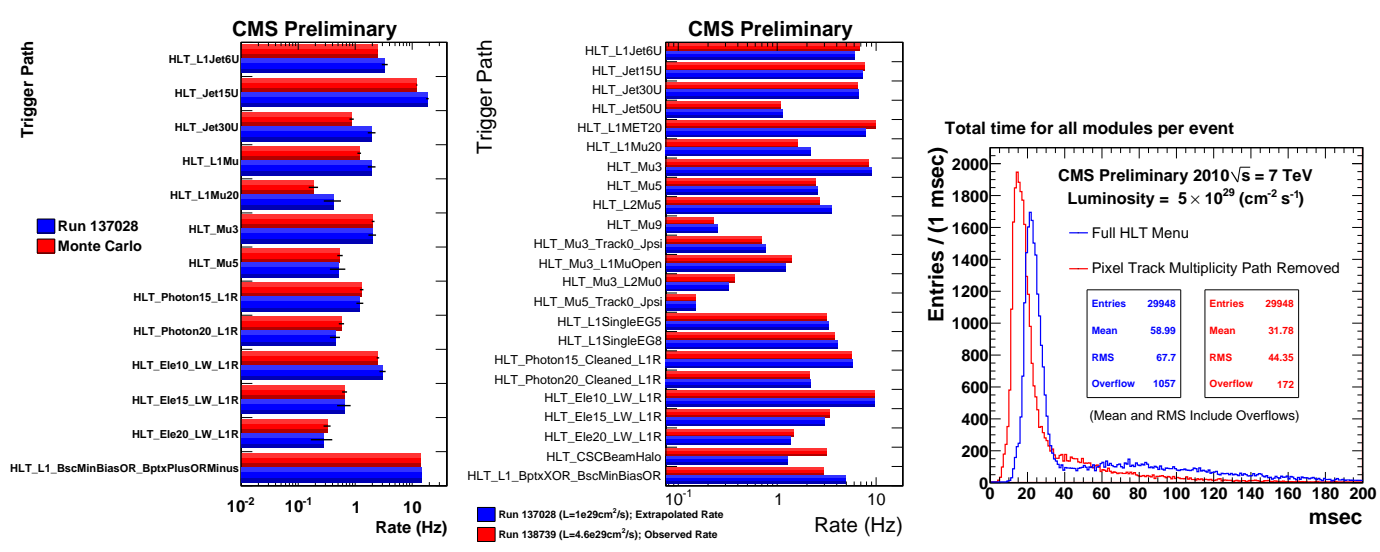

Figure 4: Rates for various HLT paths. On the left, the rates predicted from Minimum Bias MC and the rates observed in data. The rates as predicted by extrapolating the rates from data at lower instantaneous luminosities and the observed rates are shown in the center plot. The total CPU time per event taken by the HLT menu is shown on the right. For the latter, the blue distribution includes the time spent to run triggers that execute full pixel reconstruction and that are included only at start-up. The red distribution excludes these paths. The overflow bins are included in the calculation of the mean and RMS values.

\section{Conclusions}

During the start-up period of the LHC, the CMS HLT system showed excellent performance reducing the background and L1 rate optimally to the desired levels. The full understanding of the trigger rates and CPU timing allowed for a smooth first period of data taking. The efficiency curves for the electron/photon, muon, and jet/MET triggers are in general in very good shape, mostly in agreement with simulation, with discrepancies fully understood. The HLT system will keep adjusting quickly and optimally to the delivery of collisions at higher instantaneous luminosities.

\section{References}

[1] CMS Collaboration, The CMS experiment at the CERN LHC, JINST 3 (2008), no. S08004, doi:10.1088/1748-0221/3/08/S08004.

[2] CMS Collaboration, CMS High Level Trigger, CERN/LHCC 2007-021 (2007).

[3] CMS Collaboration, Performance of the CMS High Level Trigger, JINST 5 (2010) no. T03005, doi: 10.1088/1748-0221/5/03/T03005. 